\title{
Screening for mild cognitive impairment in patients with cardiovascular risk factors
}

This article was published in the following Dove Press journal:

Neuropsychiatric Disease and Treatment

\author{
Teodora Yaneva-Sirakova' \\ Latchezar Traykov ${ }^{2}$ \\ Julia Petrova ${ }^{2}$ \\ Ivan Gruev 3 \\ Dobrin Vassilev'
}

'Department of Internal Medicine, Cardiology Clinic, ${ }^{2}$ Department of Neurology, Neurology Clinic, Medical University Sofia, ${ }^{3}$ Cardiology Clinic, National Transport Hospital "Tsar Boris III", Sofia, Bulgaria

Correspondence: Teodora YanevaSirakova

Department of Internal Medicine, Cardiology Clinic, Medical University Sofia, I Georgi Sofiiski Str, 1000 Sofia Bulgaria

Tel +359889070709

Email teodora.yaneva@gmail.com
Aim: Cardiovascular risk factors are also risk factors for cognitive impairment. They have cumulative effect in target organ damage. The precise correlation between cardiovascular risk factors and cognitive impairment, as well as assessing the extent to which they may affect cognitive functioning, is difficult to ascertain in everyday clinical practice. Quick, specific, and sensitive neuropsychological tests may be useful in screening for, and the prophylaxis of, target organ damage in hypertensive patients.

Methods: We gathered full anamnesis, performed physical examination, laboratory screening and echocardiography. These variables were observed at office and home for all patients, For half of the patients, 24-hour ambulatory blood pressure monitoring and neuropsychological testing using Montreal Cognitive Assessment (MoCA), Mini Mental State Examination (MMSE), Geriatric Depression Scale, and the 4-instrumental activities of daily living scale were undertaken.

Results: For a period of 2 years, 931 patients were included after applying the inclusion and exclusion criteria. The mean age was $65.90 \pm 10.00$ years. Two hundred and sixty three patients ( 85 [32.32\%] males and 178 [67.68\%] females) were reevaluated after a mean follow-up period of 12 months (6-20 months). The mean results of MoCA and MMSE were significantly lower $(p<0.05)$ in the group of patients with poorly controlled blood pressure and cardiovascular risk factors. There was mild to intermediate negative correlation between Systematic Coronary Risk Evaluation (SCORE) and the neuropsychological tests' results.

Conclusion: Cardiovascular risk factors play an important role for the development of cognitive impairment in the eastern European population because of their high frequency and interaction. The use of easily applicable neuropsychological tests in everyday clinical practice of specialties other than neurology may help in stratifying the risk for development and progression of mild cognitive impairment in this high-risk group.

Keywords: arterial hypertension, cardiovascular risk factors, mild cognitive impairment, MoCA, pulse pressure, systolic pressure

\section{Introduction}

Arterial hypertension is considered a social problem because its morbidity is found to increase with age in a population with higher mean longevity. ${ }^{1,2}$ Together with an elevated risk for cognitive decline with advanced age, the problem becomes even more severe.

There are many longitudinal studies that suggest correlation between arterial hypertension and cognitive impairment. ${ }^{3}$ Their results, however, are diverse. ${ }^{4}$ In some of the studies high blood pressure is correlated with cognitive impairment; ${ }^{5-7}$ in others, low blood pressure is found to be a prerequisite for cognitive deterioration. ${ }^{8,9}$ Precise correlation is dependent on the age of the patient too. Blood pressure variables such as pulse pressure, systolic, diastolic pressure, early morning surge and variability may play an important role too, but the precise correlation is still unclear. ${ }^{10,11}$ It is suggested 
that some of the antihypertensive medications may have a protective effect for cognitive functioning, ${ }^{12,13}$ but the results are not definite and cannot be drawn as a group effect. In the recent years, the definition and pathogenesis of cognitive impairment have been subject to changes. Cardiovascular risk factors are emerging as an important pathogenetic factor for the development of cognitive impairment. ${ }^{11,14}$ Aging of the general population leads to higher dementia morbidity. Cardiovascular risk factors have cumulative effects and arterial hypertension has the most significant role. Thus, the brain as a target organ is subjected to the detrimental effect of arterial hypertension and other cardiovascular risk factors for a longer period of time and patients live with compromised cognitive functioning and lower quality of life for most of their lives. Dementia is the latest irreversible stage of the continuum of cognitive decline. It is correlated with significant negative impact on quality of life. Major efforts are ongoing in the field of prophylaxis of such socially important diseases as arterial hypertension and mild cognitive impairment (MCI).

Cardiovascular risk factors are also risk factors for cognitive impairment. ${ }^{14}$ They have a cumulative effect in the development of target organ damage and, in this line, dementia. ${ }^{14}$ Whitmer et al ${ }^{15}$ studied 8,845 patients between 40 and 44 years. Among them, 721 (8.2\%) patients developed dementia for the rest of their lives. Every cardiovascular risk factor (arterial hypertension, smoking, elevated cholesterol, diabetes mellitus) in the middle age elevates the risk for dementia later in life by $20 \%-40 \%$.

Patients with cardiovascular risk factors and diseases attend the cardiologists' clinic/hospital. That is why it is highly important for the cardiologist to be vigilant for any cognitive deterioration in the patient, which on the other hand can impair compliance to treatment and lead to poorer control of cardiovascular risk factors and further deterioration of cognitive functioning. Most of the diagnostic tests for MCI are not applicable in everyday clinical practice and on large scale. It is important to find specific, sensitive, and readily available tests to screen patients for cognitive impairment. Patients at risk should be defined so that they can benefit timely and fully from the screening for and the prophylaxis of cognitive impairment.

\section{Aim}

The aim of the current study is to evaluate (clinically, laboratory, and instrumentally) and to follow up patients with arterial hypertension and concomitant cardiovascular risk factors. We intend to assess their risk for the development of cognitive impairment, correlate it with the variables of blood pressure measured with different methods, and assess the role of the other cardiovascular risk factors.

\section{Methods}

\section{Inclusion criteria}

The criteria for inclusion were arterial hypertension with a history of at least 1 year (patients diagnosed with arterial hypertension or taking antihypertensive medications for $\geq 12$ months); left ventricular ejection fraction above $35 \%$; antihypertensive treatment; and regular self-measurement of brachial blood pressure.

\section{Exclusion criteria}

The exclusion criterial were acute coronary syndrome with or without ST elevation and indications for urgent coronary intervention; acute stroke; acute or chronic heart failure with ejection fraction $<35 \%$; acute kidney dysfunction or end stage chronic kidney disease and chronic dialysis; coma, spoor, or somnolence; taking antidepressants during the last 2 weeks; with impaired speech, vision, hearing; heavy head trauma; epilepsy; anemic syndrome; atrial fibrillation with suboptimal anticoagulation, verified with International Normalized Ratio testing on enrollment; poorly controlled diabetes mellitus; hypoglycemic or hyperglycemic coma; asthenoadynamic syndrome; alcoholism; diagnosed psychiatric disease, diagnosed Alzheimer or other types of dementia.

Anamnesis and a comprehensive hypertensive history were obtained for every patient. All of them underwent physical examination and basic laboratory testing. Neuropsychological tests and physical examination, as well as the education of measurement of blood pressure, were done by one and the same physician, after explaining to the patients the aim of the study. Patients were included in the study only after signing a written consent for participation in a scientific study (according to the Helsinki declaration for human research). The study was approved by the Medical Science Commission of Medical University Sofia, Bulgaria and its Ethics Committee (contract number 26 D/2011). All patients attended the cardiology clinic of a University Hospital.

\section{Methods for blood pressure measurement}

\section{Home-measured blood pressure (HMBP)}

HMBP values were gathered after educating patients on how to measure their blood pressure values properly and maintaining a diary for 3-7 days. The patients measured their blood pressure 2 times per day: 1 set of 3 measurements in the morning, after waking up, but before getting 
up from bed, and another set of measurements in the evening. We encouraged the usage of devices for brachial measurement-oscillometric, automated, or semiautomated. The target values were chosen in accordance with scientific recommendations. ${ }^{16,17}$ Blood pressure was considered controlled if the mean values were below $135 / 85 \mathrm{mmHg}$.

\section{4-Hour ambulatory blood pressure monitoring}

This was conducted with TM2430 (Boso, Germany), validated according to the requirements of the European Society of Hypertension and the British Hypertension Society. ${ }^{17,18}$ It is an oscillometric sphygmomanometer. The measurement protocol was accepted as suitable if it consisted of at least $70 \%$ valid measurements, distributed proportionally during the day and night and conducted with patients without limitations of their habitual daily activities; maintaining their regular medication regimen and duration of the recording -24 hours. The cuff was put on the nondominant hand and every patient received instructions how to behave for the apparatus to make valid measurements. Measurement intervals were set at 15 minutes during the day and at 30 minutes during the night, with passive day and night period registration (7.00 am-10.00 pm). Dipping index and blood pressure variability were automatically acquired. ${ }^{17-20}$ Some patients exhibited optimal control on home or office measurements, but still reported impaired circadian rhythm, excessive morning surge of blood pressure, or for some reason were without elevations of their blood pressure during the active day. Their risk profile is generally elevated.

\section{Office blood pressure measurement}

This was carried out as the conventional recommendations required. ${ }^{17,21,22}$ It was considered controlled to target if the mean values were below 140/90 $\mathrm{mmHg}$. We checked whether the universally accepted (for the moment) definition of target values can be applied for cognitive impairment (as a target organ damage manifestation of the brain hypertensive changes) or if we need specific brain target values. That is the reason for us to have used the accepted definitions for controlled blood pressure.

\section{Neuropsychological tests}

They were conducted in private, at least 1 hour apart and after explanation of their characteristics.

\section{Mini Mental State Examination (MMSE)}

This is the conventional neuropsychological test widely used in dementia studies. It is a validated and proven method for the diagnosis of advanced stages of cognitive impairment, but quite insensitive to the earliest phases. ${ }^{23,24}$ The cognitive domains tested in MMSE are orientation, registration, attention, and calculation (serial 7-s or spelling backwards), recall, language, and praxis (naming, repetition, 3-stage command, reading, writing, coping). The threshold for cognitive impairment is 24 points from a total of 30 . The time given for completion of the test is 5-10 minutes.

\section{Montreal Cognitive Assessment (MoCA)}

The low sensitivity of MMSE led us to the inclusion of another test - more sensitive and specific for the earliest stages of cognitive impairment. MoCA is a 10-minute compilation of neuropsychological tests aimed at evaluating frontal executive functioning and attention. ${ }^{25}$ The threshold for diagnosing cognitive impairment is 26 (out of maximal 30 points). Cognitive domains tested via MoCA are attention, concentration, executive functioning, language, memory, visual constructional, abstraction, delayed recall, and orientation skills. The test is adjusted to the education status.

\section{Geriatric Depression Scale}

Depression often coexists with dementia, but its correlation with MCI has not been studied. This test was used to study dementia patients and to explain if it was connected with earlier stages of cognitive impairment. ${ }^{26}$

\section{4-Point version of the scale for evaluating performance in} instrumental activities of daily living (4-IADL) ${ }^{27}$

Impaired daily functioning is part of dementia diagnosis. This test was used to better define patients with advanced cognitive impairment. It consists of 4 major questions. The answers to every question are assessed with $0-4$ points, depending on the stage of autonomy of the patient. A score of 0 means absolutely autonomous, 1 point - partly dependent, $\geq 2$ - with dementia risk. The questions are as follows: ability to use a telephone, to write or send a letter; to use transportation means; and to take medications alone.

Cardiovascular risk factors were defined according to the universal definition. ${ }^{28,29}$ Patients with proven coronary heart disease or diabetes mellitus type 2 , or extremely elevated cardiovascular risk factors, were considered to be under very high cardiovascular risk. In all other patients, the risk was assessed with the Systematic Coronary Risk Evaluation (SCORE) system. ${ }^{30}$

Statistical analysis was performed with SPSS version 19 (IBM Corporation, Armonk, NY, USA). The specific analysis applied was dependent on the question and the type of data. Descriptive statistics was expressed as means and standard deviation. We used Pearson correlation analysis to find 
the correlation. A nonparametric $t$-test was used to compare means. Statistics were carried out by a certified medical statistician. When comparing the mean results from neuropsychological tests between 2 groups we used the $t$-test. We checked for Sig (2-tailed) and compared it with the level of the risk for $\alpha(0.05)$ mistake. H0 was defined as "there is no statistically significant difference between the mean values of neuropsychological tests". H1 was defined as "the difference is significant, it is not accidental and is high enough to consider it significant." A 95\% confidence interval was used for acceptance of the alternative hypothesis. The number of screened patients was higher than the sample size needed as assessed with SPSS to minimize $\alpha$ and $\beta$ errors, and taking into account the frequency of both MCI and arterial hypertension in the Caucasian population. It should be noted, however, that this was the first study in an eastern European country, in which the population screened for MCI had high cardiovascular risk.

For a period of 2 years, 931 consecutive patients were included in the study with a strict consideration of the inclusion and exclusion criteria. After the initial neuropsychological screening and blood pressure assessment, the patients were divided into 2 groups, which were compared:

- with HMBP controlled to the target values - 329 $(35.34 \%), 121(13.00 \%)$ of whom were males and 208 (22.34\%) females;

- with HMBP not in the reference range - $602(64.66 \%)$, $226(24.27 \%)$ of whom were males and $376(40.39 \%)$ females.

We used "controlled" rather than "normal" blood pressure, because normal blood pressure means values below $120 / 70 \mathrm{mmHg}$, which is quite a low value. Our aim was to find "normal for the brain" values.

The groups controlled to target values (below $135 / 85 \mathrm{mmHg}$ ) versus poorly controlled blood pressure (above the target values $\geq 135 / 85 \mathrm{mmHg}$ ) were selected in order to study the difference between them on account of cognitive decline. There was no statistically significant difference between them on account of other cardiovascular risk factors, antihypertensive treatment, comorbidities. It was not possible to find a large enough age-matched (around 65 years) group without hypertension and other cardiovascular risk factors, in order to compare cognitive impairment frequency and decline. Last, but not least, this was a study from real life and these were regular patients that every cardiologist meets in their practice every day. The initial screening group was much larger than the consequent reassessment group, because we wanted to study the tendency in the frequency of cognitive impairment in hypertensive patients with comorbidities. We needed a follow-up of 200 patients to reject $\mathrm{H} 0$ with a very good level of $\alpha$ and $\beta$ errors. Once this was achieved, we concentrated on implementing our results as a standard practice in our clinic.

Two hundred and sixty three patients (85 [32.32\%] males and 178 [67.68\%] females) were followed up for a mean of 12 months (6-20 months) and were reevaluated with neuropsychological tests and various blood pressure measurement methods.

The trial was open. Both the doctor and the patient knew the results from the neuropsychological tests and discussed the possibilities for intervention toward better control of blood pressure and other cardiovascular risk factors, and follow-up of cognitive decline.

\section{Results}

In all, 347 (37.27\%) males and 584 (62.73\%) females were included in this study. $193(55.62 \%)$ males and $365(62.50 \%)$ females had cognitive impairment in the inclusion testing according to at least MoCA, if not both MoCA and MMSE.

Two hundred and sixty three (28.25\%) patients underwent reevaluation after at least 6 months: 178 (30.48\% from the included females) and 85 (24.49\% from the included men). Forty five (52.94\%) males and 132 (74.16\%) females had cognitive impairment on follow-up.

The mean age of the studied group was $65.90 \pm 10.00$ years. This was a relatively young group of patients in comparison with other similar studies. The age span was 38-92 years.

Most patients had $\geq 3$ cardiovascular risk factors (42.32\%); some had 2 cardiovascular risk factors (33.33\%); and $21.26 \%$ had only 1 isolated risk factor - arterial hypertension. In all, 209 (22.94\%) were smokers, 488 (54.28\%) had dyslipidemia, 250 (26.88\%) had diabetes mellitus type 2 , and 276 (29.68\%) were obese. The definition of cardiovascular risk was in accordance with the general recommendations. Patients with known diabetes, cardiovascular disease, or extremely elevated risk factors were considered to be at very high cardiovascular risk. All other patients were assessed with the use of SCORE for high-risk countries. From all patients with MCI, 277 (63.10\%) exhibited very high cardiovascular risk; 99 (66.44\%) high; 108 (52.68\%) intermediate, and $8(26.67 \%)$ low risk.

The mean results from MoCA were significantly $(p<0.0001)$ lower during the follow-up visit $(23.63 \pm 3.85)$ in comparison with the initial neuropsychological testing on inclusion $(24.23 \pm 3.43)$. Fifty five percent of the cases had negative change - lower MoCA results on follow-up; 
Table I Difference between neuropsychological tests' results (MMSE) on inclusion and during the follow-up visit for every given age group

\begin{tabular}{llll}
\hline $\begin{array}{l}\text { Age group } \\
\text { (years) }\end{array}$ & $\begin{array}{l}\text { MoCA } \\
\text { inclusion }\end{array}$ & $\begin{array}{l}\text { MoCA } \\
\text { follow-up }\end{array}$ & -value \\
\hline$<50$ & $26.33 \pm 4.41$ & $27.67 \pm 3.39$ & 0.158 \\
$50-59$ & $26.02 \pm 3.08$ & $25.19 \pm 3.56$ & 0.010 \\
$60-69$ & $24.87 \pm 2.90$ & $24.62 \pm 3.49$ & 0.224 \\
$70-79$ & $22.97 \pm 3.41$ & $22.22 \pm 3.76$ & 0.002 \\
$\geq 80$ & $22.76 \pm 3.24$ & $21.52 \pm 2.84$ & 0.047 \\
\hline
\end{tabular}

Abbreviations: MoCA, Montreal Cognitive Assessment; MMSE, Mini Mental State Examination.

$25.50 \%$ showed no significant change; for $19 \%$ the change was positive; and in $38(14.4 \%)$ the change was $< \pm 4 \%$ (Table 1). For the follow-up period, there was a clear tendency for deterioration of cognitive functioning that was not due to transition in another age group.

Significantly $(p<0.0001)$ lower mean MMSE values were registered during the follow-up visit (27.02 \pm 2.50$)$ than on the initial screening (27.72 \pm 2.12 ). There was no transition from one age group to another; this difference cannot be attributed to aging. The $t$-test used revealed that the difference of 0.632 between the mean values of the 2 periods of interest was statistically significant. The change of every single value in a given patient showed that in $35.6 \%$ of the patients ( 93 cases) there was no significant change between the 2 periods; in 101 $(38.70 \%)$ of the valid cases the change was small $( \pm 4 \%)$; in $52.9 \%$ the change was negative - with follow-up mean values significantly lower than those of the initial neuropsychological test; the follow-up visit test results were better for only $11.5 \%$ of the cases (Table 2). Consequently, the results deteriorated both between the age groups and between the initial and follow-up visit in every single age group.

Neuropsychological results for MoCA subtests are presented in Table 3.

Table 4 shows the mean results of MoCA subtests, in which we registered a significant difference between the

Table 2 Difference between neuropsychological tests results (MMSE) on inclusion and during the follow-up visit for every given age group

\begin{tabular}{llll}
\hline $\begin{array}{l}\text { Age group } \\
\text { (years) }\end{array}$ & $\begin{array}{l}\text { MMSE } \\
\text { (initial) } \pm \text { SD }\end{array}$ & $\begin{array}{l}\text { MMSE } \\
\text { (follow-up) } \pm \text { SD }\end{array}$ & p-value \\
\hline$<50$ & $28.67 \pm 1.97$ & $28.67 \pm 2.16$ & $\mathrm{I}$ \\
$50-59$ & $28.46 \pm 1.66$ & $28.10 \pm 2.23$ & 0.162 \\
$60-69$ & $28.19 \pm 1.78$ & $27.87 \pm 2.12$ & 0.008 \\
$70-79$ & $27.23 \pm 2.23$ & $26.30 \pm 2.46$ & 0.000 \\
$\geq 80$ & $26.05 \pm 2.48$ & $24.76 \pm 2.21$ & 0.001 \\
\hline
\end{tabular}

Abbreviations: MMSE, Mini Mental State Examination; SD, standard deviation.
Table 3 Mean results from the MoCA subtests and the percentage of patients with poor results

\begin{tabular}{|c|c|c|c|c|}
\hline \multirow[t]{2}{*}{$\begin{array}{l}\text { Neuropsychological } \\
\text { test }\end{array}$} & \multicolumn{2}{|c|}{ Mean results } & \multicolumn{2}{|c|}{$\begin{array}{l}\% \text { of patients with } \\
\text { poor results }\end{array}$} \\
\hline & Initial & $\begin{array}{l}\text { On } \\
\text { follow-up }\end{array}$ & Initial & $\begin{array}{l}\text { On } \\
\text { follow-up }\end{array}$ \\
\hline TMT-B & $0.22 \pm 0.41$ & $0.22 \pm 0.42$ & 77.93 & 77.61 \\
\hline Cube drawing & $0.64 \pm 0.48$ & $0.56 \pm 0.52$ & 36.17 & 44.79 \\
\hline Clock drawing & $2.42 \pm 0.84$ & $2.23 \pm 0.93$ & 37.89 & 47.10 \\
\hline Naming & $2.93 \pm 0.29$ & $2.88 \pm 0.35$ & 5.81 & 11.58 \\
\hline Forward digit span & $0.87 \pm 0.38$ & $0.85 \pm 0.36$ & 14.64 & 15.06 \\
\hline Backward digit span & $0.88 \pm 0.32$ & $0.86 \pm 0.35$ & 11.73 & 13.9 \\
\hline Vigilance & $0.99 \pm 0.09$ & $0.99 \pm 0.09$ & 0.75 & 0.72 \\
\hline Serial 7-s & $2.73 \pm 0.65$ & $2.64 \pm 0.70$ & 17.44 & 23.94 \\
\hline Sentence repetition & $1.63 \pm 0.64$ & $1.64 \pm 0.62$ & 28.31 & 28.57 \\
\hline Fluency & $0.52 \pm 0.50$ & $0.49 \pm 0.50$ & 48.65 & 50.58 \\
\hline Abstraction & $1.01 \pm 0.79$ & $0.84 \pm 0.78$ & 68.03 & 76.06 \\
\hline Late recall & $2.74 \pm 1.49$ & $2.80 \pm 1.48$ & 87.51 & 84.55 \\
\hline Orientation & $6.00 \pm 0.00$ & $6.00 \pm 0.00$ & 0 & 0 \\
\hline
\end{tabular}

Abbreviations: MoCA, Montreal Cognitive Assessment; TMT-B, trail making test part B.

groups of patients with controlled and poorly controlled hypertension. The neuropsychological domains that were predominantly affected were memory - short memory (digits forward test), working memory (backwards digit span) - and executive functioning (trial making test $\mathrm{B}$, cube drawing, clock drawing, naming).

Figure 1 gives the comparison between the neuropsychological test results of patients with controlled target value blood pressure and those with suboptimally controlled blood pressure. The correlation analysis between SCORE and neuropsychological tests results is given on Table 5 . Table 6 shows the comparison of MoCA and MMSE results between the groups of patients with a certain cardio-vascular risk factor and those without it.

Table 4 Difference between mean results from the MoCA subtests in patients with controlled and poorly controlled blood pressure

\begin{tabular}{llll}
\hline $\begin{array}{l}\text { Neuropsychological } \\
\text { test }\end{array}$ & $<\mathbf{1 3 5 / 8 5} \mathbf{~ m m H g} \geq \mathbf{1 3 5 / 8 5} \mathbf{~ m m H g}$ & $\mathbf{p}$-value \\
\hline TMT-B inclusion & $0.27 \pm 0.44$ & $0.20 \pm 0.40$ & 0.019 \\
Cube inclusion & $0.73 \pm 0.45$ & $0.69 \pm 0.49$ & $<0.000 \mathrm{I}$ \\
Clock inclusion & $2.55 \pm 0.78$ & $2.35 \pm 0.86$ & $<0.000 \mathrm{I}$ \\
Naming inclusion & $2.96 \pm 0.2 \mathrm{I}$ & $2.92 \pm 0.33$ & 0.024 \\
Forward digit span & $0.88 \pm 0.32$ & $0.86 \pm 0.40$ & $\mathrm{~ns}$ \\
inclusion & & & \\
$\begin{array}{l}\text { Backward digit span } \\
\text { inclusion }\end{array}$ & $0.91 \pm 0.28$ & $0.87 \pm 0.34$ & 0.032 \\
\hline
\end{tabular}

Abbreviations: MoCA, Montreal Cognitive Assessment; ns, not significant; TMT-B, trail making test part $B$. 


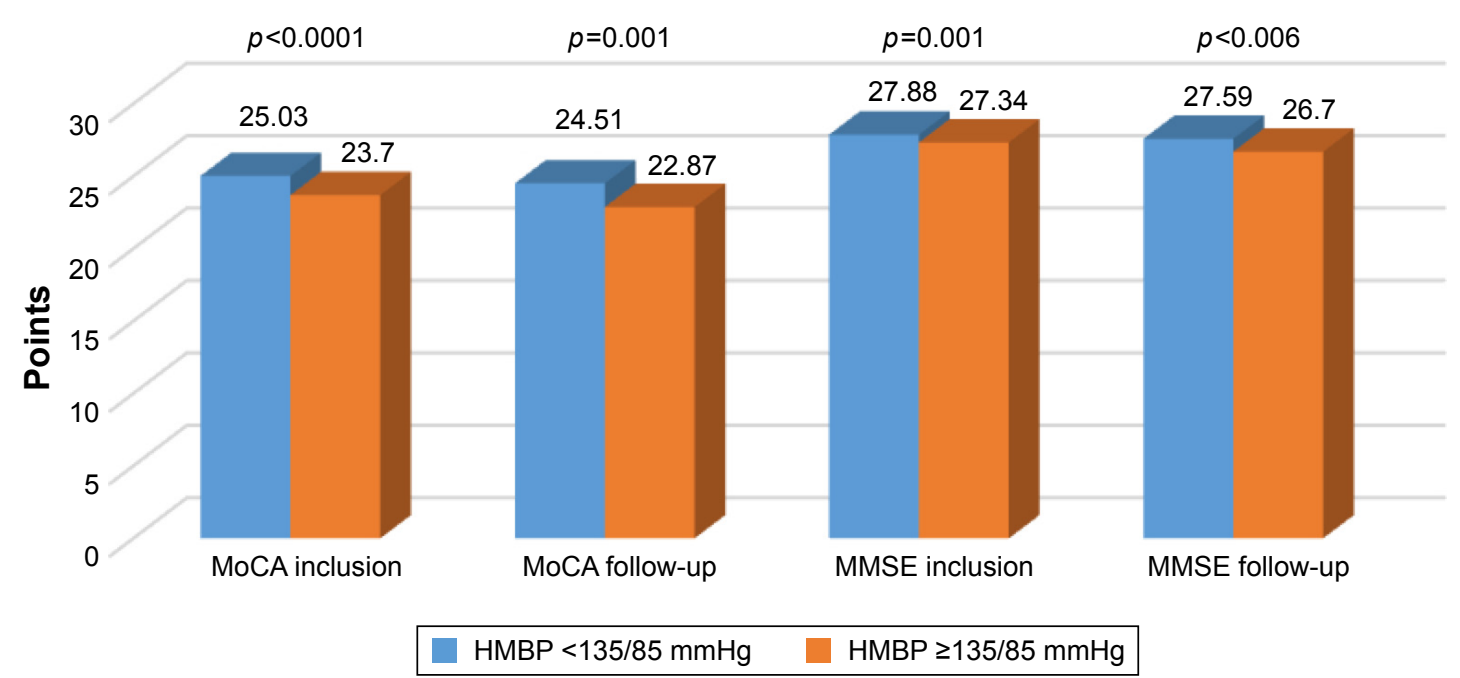

Figure I Comparison between the neuropsychological tests results of patients with controlled target values $(<135 / 85 \mathrm{mmHg})$ and suboptimally controlled blood pressure. Note: The mean neuropsychological results were significantly $(p<0.005)$ lower in the follow-up visit in comparison with the initial results.

Abbreviations: MoCA, Montreal Cognitive Assessment; MMSE, Mini Mental State Examination; HMBP, home-measured blood pressure.

Thirty two (82.05\%) patients with depression simultaneously had MCI. The mean values of MoCA and MMSE in patients with depression were $22.23 \pm 3.69$ and $26.10 \pm 3.3$, respectively. They were significantly lower than the corresponding mean values in patients without depression, $24.26 \pm 3.52$ with $p<0.0001$ for MoCA and 27.61 \pm 2.36 with $p=0.008$ for MMSE.

The 4-IADL scale was used for assessment of the ability of the patients to perform everyday activities. All patients with severely impaired daily activities had cognitive impairment. From the group of patients who were partially dependent on caregivers' help, 21 (80.77\%) had MCI.

\section{Discussion}

The results from this study are confirmatory for the Caucasian population, but still of considerable significance for the Bulgarian population, because the problem is evolving. Many studies have addressed the same problem, but a universal answer has not been obtained yet. ${ }^{31-33}$ There is even a consensus statement for vascular contributions to cognitive

Table 5 Correlation between SCORE system for cardiovascular risk assessment and results from the neuropsychological tests

\begin{tabular}{lllll}
\hline Variable & $\begin{array}{l}\text { MoCA } \\
\text { inclusion }\end{array}$ & $\begin{array}{l}\text { MoCA } \\
\text { follow-up }\end{array}$ & $\begin{array}{l}\text { MMSE } \\
\text { inclusion }\end{array}$ & $\begin{array}{l}\text { MMSE } \\
\text { follow-up }\end{array}$ \\
\hline$r$ & $-0.156^{* *}$ & $-0.161^{*}$ & $-0.147^{* *}$ & -0.121 \\
$p$-value & $<0.0001$ & 0.011 & $<0.0001$ & 0.059 \\
$\mathrm{n}$ & 845 & 247 & 845 & 245 \\
\hline
\end{tabular}

Note: ${ }^{* *}$ Confidence interval $99 \%$, *onfidence interval $95 \%, r-$ Pearson coefficient, $\mathrm{n}$ - number.

Abbreviations: MoCA, Montreal Cognitive Assessment; MMSE, Mini Mental State Examination; SCORE, Systematic Coronary Risk Evaluation. impairment. ${ }^{10}$ However, this is a study from everyday clinical practice. Its advantage is that the patients do not have only a single isolated risk factor, as in most studies, but real life patients, with cumulative risk factors and vascular diseases. This is the first study of the type in Bulgaria, the first to correlate SCORE with cognitive decline - to use a cardiovascular assessment score for assessment of cognitive decline. The results impart value to everyday clinical practice. It formed the basis to develop a screening process for cognitive impairment in patients usually followed by cardiologists; if not screened in this manner, the patients would escape neuropsychological assessment, which can postpone diagnosis of MCI.

The higher number of female patients can be explained by 2 facts: according to European Medicines Agency, women are not adequately presented in trials concerning arterial hypertension; women have greater longevity and their relative number is higher in the advanced age population. ${ }^{34}$

In this research, $42.32 \%$ of the studied group includes patients with $\geq 3$ cardiovascular risk factors; $53.34 \%$ with very high cardiovascular risk. Patients with arterial hypertension and cardiovascular risk factors have elevated morbidity from MCI. In smokers, patients with diabetes mellitus, and dyslipidemia the proportion of newly developed (between the 2 visits) cognitive impairment is very high (40.62\%, 53.57\%, and 40.98\%, respectively). Patients with diabetes mellitus, smokers, or those who were obese had significantly lower mean results from the neuropsychological tests. The association between MCI and every cardiovascular risk factor posed the question of whether the 
Table 6 Results from the neuropsychological tests in patients with different cardiovascular risk factors

\begin{tabular}{|c|c|c|c|c|c|c|}
\hline \multirow[t]{2}{*}{ Variable } & \multicolumn{2}{|c|}{ MoCA inclusion (mean \pm SD) } & \multirow[t]{2}{*}{$p$-value } & \multicolumn{2}{|c|}{ MMSE inclusion (mean \pm SD) } & \multirow[t]{2}{*}{$p$-value } \\
\hline & With RF & Without RF & & With RF & Without RF & \\
\hline Dyslipidemia & $23.21 \pm 3.45$ & $24.33 \pm 3.54$ & 0.001 & $26.6 I \pm 2.84$ & $27.69 \pm 2.33$ & $<0.0001$ \\
\hline Diabetes mellitus type 2 & $23.59 \pm 3.82$ & $24.40 \pm 3.43$ & 0.003 & $27.29 \pm 2.47$ & $27.64 \pm 2.41$ & 0.047 \\
\hline Smoking & $24.02 \pm 3.54$ & $25.03 \pm 3.38$ & 0.001 & $27.43 \pm 2.51$ & $28.06 \pm 2.06$ & $<0.000$ I \\
\hline Obesity & $24.08 \pm 3.56$ & $24.44 \pm 3.53$ & ns & $27.42 \pm 2.48$ & $27.85 \pm 2.27$ & 0.010 \\
\hline
\end{tabular}

Abbreviations: MoCA, Montreal Cognitive Assessment; MMSE, Mini Mental State Examination; ns, not significant; SD, standard deviation; RF, risk factor.

assessment of cardiovascular risk can be used as a predictor for the development and progression of MCI. We were able to demonstrate a mild negative correlation between SCORE and the results from the neuropsychological tests. The higher the SCORE risk, the lower the neuropsychological results. One potential explanation for the low strength of the correlation is the fact that the risk factors that are incorporated in SCORE are only part of the risk factors for MCI. However, it can be used as a screening tool in patients with cardiovascular risk factors to guide screening for MCI with neuropsychological tests.

Hyperglycemia is correlated with oxidative stress and glucose mediated disruption of small vessels..$^{35}$ The dysfunction of insulin degrading enzyme leads to hyperinsulinemia and $\beta$-amyloid accumulation. ${ }^{36} \mathrm{MCI}$ in diabetic people is correlated with earlier morbidity, longer history of diabetes, and poorer diabetic control. ${ }^{37}$ There are some suggestions that Alzheimer's disease is a form of diabetes, a kind of "type 3 " diabetes. ${ }^{38}$ This thesis is supported by the results from studies with peroxisome proliferator-activated receptor agonists or intranasal insulin in patients with Alzheimer's disease ${ }^{39}$ which register an improvement of the cognitive status. ${ }^{39,40}$ In our study group, patients with diabetes showed significantly lower neuropsychological results than those without diabetes.

We found a significant difference $(p<0.05)$ in the mean values of the neuropsychological tests (Table 6) between the groups of hypertensive patients with and without dyslipidemia. Dyslipidemia is associated with vascular remodeling, change pulsatility, and permeability, that lead to local hypoxia and oxidative stress, as well as a proinflammatory state. That may be the potential explanation of the lower neuropsychological test results in patients with dyslipidemia. A separate analysis on the topic of the study group showed a protective effect of statins on cognitive decline. These results are supported by other trials. ${ }^{41,42}$

Hypercholesterolemia in middle age is a risk factor for MCI in advance age. ${ }^{15}$ In many observational studies, a positive correlation is observed between the treatment with statins and the progression of MCI and primarily Alzheimer's disease. ${ }^{42}$ However, results from 2 large randomized studies HPS $^{43}$ and PROSPER ${ }^{44}$ - do not prove any positive effect on $\mathrm{MCI}$ from the treatment with statins. On the contrary, another study shows that statins have multiple positive effects only in patients with dementia, but not in patients with $\mathrm{MCI} .{ }^{45}$ In our study, the correlation between MCI and dyslipidemia is significant only with MMSE and advanced MCI. This can be explained by the fact that the majority of patients were on statins at the time of testing. As the precise correlation between statin therapy and MCI is not clear, further studies in this field are needed.

Smoking doubles the risk of dementia, including the risk of Alzheimer's disease in advanced age. ${ }^{46,47}$ The results for 26,374 patients demonstrate this in a meta-analysis ${ }^{47}$ of 19 prospective trials with a minimal follow-up period of 12 months. The smokers showed an elevated risk for the development of dementia and the progression of cognitive impairment in our group too.

Obese, middle-aged patients show an elevated risk for the development of dementia in advanced age. ${ }^{48}$ On the contrary, obesity in advanced age is not invariably correlated with cognitive impairment. ${ }^{49}$ Patients with extremely low body mass also demonstrate elevated risk for cognitive impairment. ${ }^{50}$ Such results have provoked, in the literature, the thesis for the so-called "obesity paradox" ${ }^{51}$ Central obesity leads to elevated inflammatory markers. Lipid tissue secretes inflammatory cytokines and growth hormones (leptin, interleukin-6, C-reactive protein). These neurohormones are correlated with cognitive functioning. ${ }^{52}$ The mean age of our study group is lower than the mean age in the majority of other trials for cognitive impairment. This can explain the lower MMSE results in obese patients.

Dementia is usually accompanied with depression. Cardiovascular diseases are also correlated with depression. It is important to know if there are people with depression in this group of patients with cardiovascular risk factors and diseases and MCI. Depression may undermine compliance to treatment and further impair blood pressure 


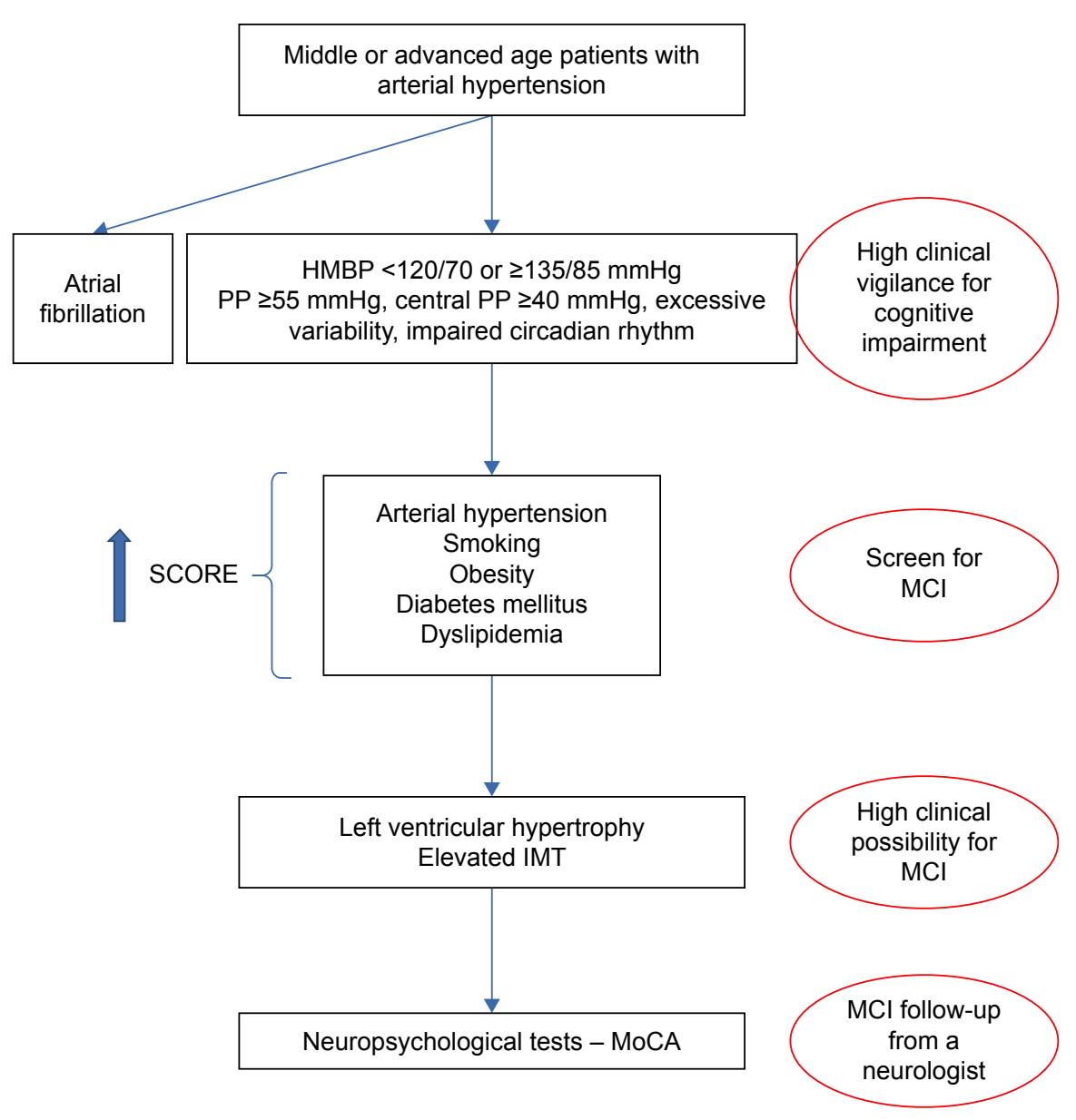

Figure 2 Flowchart for screening for $\mathrm{MCl}$ in everyday clinical practice in hypertensive patients with cardiovascular disease.

Abbreviations: MoCA, Montreal Cognitive Assessment; $\mathrm{MCl}$, mild cognitive impairment; HMBP, home-measured blood pressure; SCORE, Systematic Coronary Risk Evaluation; Pts, patients; IMT, intima-media-thickness; PP, pulse pressure.

control. The correlation between MCI in patients with cardiovascular risk factors and depression is proven in our study.

\section{Conclusion}

This study showed that $21.74 \%$ of the patients in the age group $<50$ years, $40.28 \%$ in the age group $50-60$ years, and $58.22 \%$ in the age group $60-70$ years have clinical manifestations of MCI. The frequency of MCI in patients with arterial hypertension is $59.93 \%$ for the entire group and $68 \%$ in the group of patients with poorly controlled HMBP. The frequency of MCI in this high-risk group is very high, which is a good reason for screening and timely treatment. Patients with poor blood pressure control show lower $(p<0.05)$ results from the neuropsychological tests and should receive special follow-up and interdisciplinary control.

SCORE can be used for initial screening for the risk of MCI in patients with arterial hypertension and variable cardiovascular risk factors.
On the basis of our results we developed a flowchart for screening for MCI in everyday clinical practice in hypertensive patients with cardiovascular disease (Figure 2).

There are patients with depression in the group with cardiovascular risk factors and cognitive impairment. Autonomy is not generally affected. We expect that, at this early stage of cognitive impairment, screening and follow-up will be beneficial for timely diagnosis and prophylaxis of deterioration, because autonomy is still spared and only a minority of the patients have depression that can possibly impair their treatment compliance.

Every cardiovascular risk factor affects target organs. Their combination, however, leads to a cumulative negative effect. In everyday practice, cardiovascular risk factors are almost never isolated. This should be always considered. Doctors should conduct screening for MCI in patients with multiple cardiovascular risk factors. The prophylaxis of target organ damage (MCI too) should start early enough and be aimed at all of the risk factors simultaneously. 


\section{Disclosure}

The authors report no conflicts of interest in this work.

\section{References}

1. James P, Oparil S, Carter B, et al. 2014 Evidence-based guideline for the management of high blood pressure in adults: report from the panel members appointed to the Eight Joint National Committee (JNC 8). JAMA. 2014;311(5):507-520.

2. Touyz RM, Dominiczak A. Hypertension guidelines. Is it time to reappraise blood pressure thresholds and targets? Hypertension. 2016; 67:688-689.

3. Wysocki M, Luo X, Schmeidler J, et al. Hypertension is associated with cognitive decline in elderly people at high risk for dementia. Am J Geriatr Psychiatry. 2012;20(2):179-187.

4. Igase M, Kohara K, Miki T. The association between hypertension and dementia in the elderly. Int J Hypertens. 2012;2012:320648.

5. Skoog I, Lernfelt B, Landahl S, et al. 15-Year longitudinal study of blood pressure and dementia. Lancet. 1996;347(9009):1141-1145.

6. Launer L, Ross G, Petrovitch H, et al. Midlife blood pressure and dementia: the Honolulu-Asia aging study. Neurobiol Aging. 2000;21(1): $49-55$.

7. Kivipelto M, Helkala E, Laakso M, et al. Midlife vascular risk factors and Alzheimer's disease in later life: longitudinal, population based study. BMJ. 2001;322(7300):1447-1451.

8. Moretti R, Torre P, Antonello R, et al. Risk factors for vascular dementia: hypotension as a key point. Vasc Health Risk Manag. 2008; 4(2):395-402.

9. Verghese J, Lipton R, Hall C, et al. Low blood pressure and the risk of dementia in very old individuals. Neurology. 2003;61(12): 1667-1672.

10. Gorelick P, Scuteri A, Black S, et al. Vascular contributions to cognitive impairment and dementia: a statement for healthcare professionals from the American Heart Association/American Stroke Association. Stroke. 2011;42(9):2672-2713.

11. Gunstad J, Keary TA, Pappas A, et al. Blood pressure and cognitive function in older adults with cardiovascular disease. Int $J$ Neurosci. 2009;119(12):2228-2242.

12. Marpillat L, Macquin-Mavier I, Tropeano AL, et al. Antihypertensive classes, cognitive decline and incidence of dementia: a network metaanalysis. J Hypertens. 2013;31(6):1073-1082.

13. Duron E, Hanon O. Antihypertensive treatments, cognitive decline, and dementia. J Alzheimers Dis. 2010;20(3):903-914.

14. Kivipelto M, Nqandu T, Fratiglioni L, et al. Obesity and vascular risk factors at midlife and the risk of dementia and Alzheimer disease. Arch Neurol. 2005;62(10):1556-1560.

15. Whitmer R, Sidney S, Selby J, et al. Midlife cardiovascular risk factors and risk of dementia in late life. Neurology. 2005;64:277-281.

16. Mancia G, De Backer G, Dominiczak A, et al. 2007 Guidelines for the management of arterial hypertension. Eur Heart J. 2007;28: 1462-1536.

17. O'Brien E, Asmar R, Beilin L, et al; European Society of Hypertension Working Group on Blood Pressure Monitoring. Practice guidelines of the European Society of Hypertension for clinic, ambulatory and self blood pressure measurement. J Hypertens. 2005;23:697-701.

18. O’Brien E, Atkins N, Stergiou G, et al. European Society of Hypertension International Protocol revision 2010 for the validation of blood pressure measuring devices in adults. Blood Press Monit. 2010;15(1): 23-38.

19. Ben-Dov I, Ben-Arie L, Mekler J, Bursztyn M. Normal ambulatory blood pressure: a clinical-practice based analysis of recent American Heart Association recommendations. Am J Med. 2006;119:69.

20. Mancia G, Laurent S, Agabiti-Rose E, et al. Reappraisal of European guidelines on hypertension management: a European Society of Hypertension Task Force document. J Hypertens. 2009;27: 2121-2158.
21. Mancia G, Facchetti R, Bombelli M, Grassi G, Sega R. Long-term risk of mortality associated with selective and combined elevation in office, home, and ambulatory blood pressure. Hypertension. 2006;47: 846-853.

22. Pickering T, Hall J, Appel L, et al. Recommendations for blood pressure measurement in humans and experimental animals. Part 1: blood pressure measurement in humans: a statement for professionals from the Subcommittee of Professional and Public Education of the American Heart Association Council on High Blood Pressure Research. Hypertension. 2005;45(1):142-161.

23. Bravo G, Hebert R. Age- and education-specific reference values for the Mini-Mental and modified Mini-Mental State Examinations derived from a non-demented elderly population. Int $J$ Geriatr Psychiatry. 1997;12(10):1008-1018.

24. O-Bryant S, Humphreys J, Smith G, et al. Detecting dementia with the Mini-Mental State Examination (MMSE) in highly educated individuals. Arch Neurol. 2008;65(7):963-967.

25. MoCA. Available from: www.mocatest.org/paper-tests/moca-test-full/. Accessed November 1, 2017.

26. Almeida O, Almeida SH. Short versions of the geriatric depression scale: a study of their validity for the diagnosis of a major depressive episode according to ICD-10 and DSM-IV. Int J Geriatr Psychiatry. 1999;14: 858-865.

27. Hancock P, Larner AJ. The diagnosis of dementia: diagnostic accuracy of an instrument measuring activities of daily living in a clinic-based population. Dement Geriatr Cogn Disord. 2007;23:133-139.

28. Pearson TH, Blair S, Daniels S, et al. AHA guidelines for primary prevention of cardiovascular disease and stroke: 2002 update. Circulation. 2002;106:388-391.

29. Kotseva K, Wood D, DeBacquer D, et al. EUROASPIRE IV: a European Society of Cardiology survey on the lifestyle, risk factor and therapeutic management of coronary patients from 24 European countries. Eur $J$ Prev Cardiol. 2016;23:636-648.

30. Perk J, de Backer G, Gohlke H, et al. European guidelines on cardiovascular disease prevention in clinical practice (version 2012). Eur Heart J. 2012;33:1635-1701.

31. Elias M, Wolf P, D’Agostino R, Cobb J, White LR. Untreated blood pressure level is inversely related to cognitive functioning: the Framingham Study. Am J Epidemiol. 1993;138(6):353-364.

32. Feldman H, Levy A, Hsiung G, et al. A Canadian cohort of cognitive impairment and related dementias (ASSORD): study methods and baseline results. Neuroepidemiology. 2003;22:265-274.

33. Goldstein I, Bartzokis G, Hance D, Shapiro D. Relationship between blood pressure and subcortical lesions in healthy elderly people. Stroke. 1998;29(4):765-772.

34. National Statistical Institute. Health. Available from: www.nsi.bg/en/ content $/ 5553 /$ health. Accessed November 1, 2017.

35. Brownlee M. Biochemistry and molecular cell biology of diabetic complications. Nature. 2001;414:813-820.

36. Farris W, Mansourian S, Chang Y, et al. Insulin-degrading enzyme regulates the levels of insulin, amyloid beta-protein, and the beta-amyloid precursor protein intracellular domain in vivo. Proc Natl Acad Sci USA. 2003; 100:4162-4167.

37. Roberts R, Geda Y, Knopman D. Association of duration and severity of diabetes mellitus with mild cognitive impairment. Arch Neurol. 2008;65(8):1066-1073.

38. de la Monte S, Wands J. Alzheimer's disease is type 3 diabetes evidence reviewed. J Diabetes Sci Technol. 2008;2(6):1101-1113.

39. Landreth G. Therapeutic use of agonists of the nuclear receptor PPARgamma in Alzheimer's disease. Curr Alzheimer Res. 2007;4(2): 159-164.

40. Reger M, Watson G, Frey WH 2nd, et al. Effects of intranasal insulin on cognition in memory-impaired older adults: modulation by APOE genotype. Neurobiol Aging. 2006;27(3):451-458.

41. Geifman N, Brinton R, Kennedy R, Schneider LS, Butte AJ. Evidence for benefit of statins to modify cognitive decline and risk in Alzheimer's disease. Alzheimers Res Ther. 2017;9(1):10. 
42. Wolozin B, Kellman W, Ruosseau P, Celesia G, Siegel G. Decreased prevalence of Alzheimer disease associated with 3-hydroxy-3-methyglutaryl coenzyme A reductase inhibitors. Arch Neurol. 2000;57:1439-1443.

43. Heart Protection Study Collaborative Group. MRC/BHF Heart Protection Study of cholesterol lowering with simvastatin in 20,536 high-risk individuals: a randomized placebo-controlled trial. Lancet. 2002; 360:7-22.

44. Shepherd J, Blauw G, Murphy M, et al. Pravastatin in elderly individuals at risk of vascular disease (PROSPER): a randomised controlled trial. Lancet. 2002;360:1623-1630.

45. Beydoun M, Beason-Held L, Kitner-Triol MH, et al. Statins and serum cholesterol's associations with incident dementia and mild cognitive impairment. J Epidemiol Community Health. 2011;65:949-957.

46. Ott A, Slooter A, Hofman A, et al. Smoking and risk of dementia and Alzheimer's disease in a population-based cohort study: the Rotterdam Study. Lancet. 1998;351(9119):1840-1843.

47. Anstey K, von Sanden CH, Salim A, O’Keamey R. Smoking as risk factor for dementia and cognitive decline: a meta-analysis of prospective studies. Am J Epidemiol. 2007;166:367-378.
48. Xu W, Atti A, Gatz M, Pedersen N, Johansson B, Fratiglioni L. Midlife overweight and obesity increase late-life dementia risk: a populationbased twin study. Neurology. 2011;76(18):1568-1574.

49. Dahl A, Löppönen M, Isoaho R, Berg S, Kivela S. Overweight and obesity in old age are not associated with greater dementia risk. $J \mathrm{Am}$ Geriatr Soc. 2008;56(12):2261-2266.

50. Fitzpatrick A, Kuller L, Lopez O, et al. Midlife and late-life obesity and the risk of dementia: cardiovascular health study. Arch Neurol. 2009;66(3):336-342.

51. Beckett N, Pedditzi E, Peters R. Dementia and the obesity paradox. Alzheimer's Dement. 2011;7(4):e8.

52. Misiak B, Leszek J, Kiejna A. Metabolic syndrome, mild cognitive impairment and Alzheimer's disease - the emerging role of systemic low-grade inflammation and adiposity. Brain Res Bull. 2012;89(3-4): 144-149.
Neuropsychiatric Disease and Treatment

\section{Publish your work in this journal}

Neuropsychiatric Disease and Treatment is an international, peerreviewed journal of clinical therapeutics and pharmacology focusing on concise rapid reporting of clinical or pre-clinical studies on a range of neuropsychiatric and neurological disorders. This journal is indexed on PubMed Central, the 'PsycINFO' database and CAS,

\section{Dovepress}

and is the official journal of The International Neuropsychiatric Association (INA). The manuscript management system is completely online and includes a very quick and fair peer-review system, which is all easy to use. Visit http://www.dovepress.com/testimonials.php to read real quotes from published authors. 